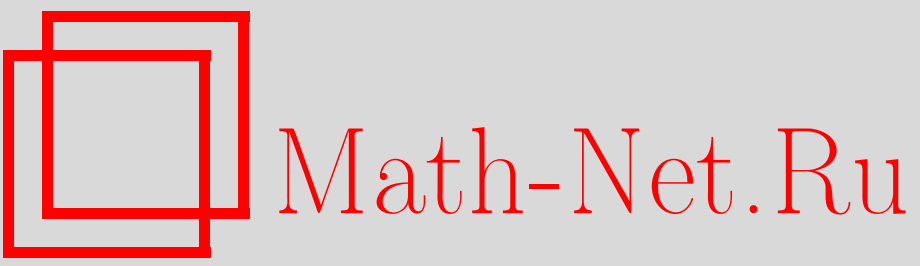

В. Е. Тарасов, Квантовые диссипативные системы. IV. Аналоги алгебры Ли и группы Ли, ТМФ, 1997, том 110, номер 2, 214-227

DOI: https://doi.org/10.4213/tmf962

Использование Общероссийского математического портала Math-Net.Ru подразумевает, что вы прочитали и согласны с пользовательским соглашением

http://www.mathnet.ru/rus/agreement

Параметры загрузки:

IP: 54.210 .77 .194

26 апреля 2023 г., 15:50:01 
ТЕОРЕТИЧЕСКАЯ

И МАТЕМАТИЧЕСКАЯ

ФИЗИКА

Том 110, № 2

февраль, 1997

B. Е. Тарасов*

\title{
КВАНТОВЫЕ ДИССИПАТИВНЫЕ СИСТЕМЫ. IV. АНАЛОГИ АЛГЕБРЫ ЛИ И ГРУППЫ ЛИ
}

\begin{abstract}
Требование самосогласованности квантового описания диссипативных систем приводит к необходимости выхода за рамки алгебр Ли и групп Ли, т.е. к использованию нелиевых алгебр (алгебр, в которых не выполняется тождество Якоби) и аналитических квазигрупп (неассоциативного обобщения групп). В данной работе показывается, что аналогами алгебры Ли и группы Ли для квантовых диссипативных систем являются коммутантно лиева алгебра (антикоммутативная алгебра, коммутант которой является лиевой подалгеброй) и аналитическая коммутантно ассоциативная лупа (лупа, коммутант которой является ассоциативной подлупой (группой)). Доказывается, что касательная алгебра аналитической коммутантно ассоциативной лупы с обратимостью (лупы Валя) является коммутантно лиевой алгеброй (алгеброй Валя). Рассматриваются примеры коммутантно лиевых алгебр.
\end{abstract}

\section{1. ВВЕДЕНИЕ}

В квантовой механике, описывающей недиссипативные (гамильтоновы) системы, используется пара, состоящая из алгебры Ли и аналитической группы (группы Ли). Члены этой пары связаны между собой теоремами Ли $[1,2]$.

Для непротиворечивости описания квантовой эволюции диссипативных (негамильтоновых) систем необходимо выйти за рамки данной пары [3]. Следовательно, необходимо использовать антикоммутативную нелиеву алгебру (алгебру, в которой не выполняется тождество Якоби) и аналитическую неассоциативную квазигруппу, или лупу [2, 4].

K сожалению, алгебры и лупы, необходимые для квантового описания диссипативных систем, не принадлежат к хорошо изученным алгебрам и лупам [5-7]. В данной четвертой части мы показываем, что искомой парой являются алгебра, коммутант которой - лиева подалгебра (алгебра Валя), и аналитическая лупа, коммутант которой ассоциативная подлупа (группа). Доказывается аналог теоремы Ли, связывающий данную аналитическую лупу с алгеброй Валя. Приводятся примеры нелиевой алгебры с лиевым коммутантом. Среди них обобшенная алгебра Гейзенберга-Вейля, предложенная в [3] для описания квантовых диссипативных систем, обобшение алгебры Пуассона дифференциальных 1-форм и векторных полей, а также алгебра бесконечных матрищ, коммутаторы которых являются ассоциативными матрицами.

\footnotetext{
*Научно-исследовательский институт ядерной физики Московского государственного университета, tarasov@theory.npi.msu.su
} 


\section{2. КОММУТАНТНО ЛИЕВЫ АЛГЕБРЫ}

2.1. Алгебра Валя, коммутант и коммутантно лиева алгебра. Введем следуюшее

ОПРЕДЕЛЕниЕ 2.1. Алгебру $B$ назовем алгеброй Валя, если операция умножения удовлетворяет

1) условию антикоммутативности: $x x=0$,

2 мягкому тождеству Якоби: $J(x y, z p, q l)=0$, где $J(x, y, z)=(x y) z+(y z) x+(z x) y-$ якобиан элементов алгебры.

Видно, что любая алгебра Ли, задаваемая соотношениями $x^{2}=0, J(x, y, z)=0$, является алгеброй Валя. Всякая бинарно лиева алгебра [2], т.е. алгебра, операция умножения которой удовлетворяет условиям $x^{2}=0, J(x, y, x y)=0$, также является алгеброй Валя.

Пусть $A$-некоторая неассоциативная алгебра. Введем билинейную операцию $[x, y]=$ $x y-y x$, назьваемую коммутатором. В результате имеем коммутаторную алгебру $A^{(-)}$, ассоциированную с алгеброй $A$. Для коммутаторной алгебры условие антикоммутативности и мягкое тождество Якоби реализуются в виде

1) $[x, x]=0$,

2) $J[[x, y],[z, p],[q, l]]=0$, где $J[x, y, z]=[[x, y], z]+[[y, z], x]+[[z, x], y]$.

Пусть, далее, $g=A^{(-)}$- коммутаторная алгебра, ассоциированная с некоторой алгеброй $A$. В коммутаторной алгебре можно определить понятие коммутанта [2].

ОПРЕДЕЛЕНИЕ 2.2. Коммутантом алгебры $g$ называется подпространство $[g, g]=g^{\prime}$, порожденное всеми коммутаторами $[x, y]$, где $x, y \in g$.

ОПрЕДЕЛЕНИЕ 2.3. Кратные коммутанты $g^{(k)}, k=0,1,2, \ldots$, алгебры $g$ определяются по индуктивному правилу: $g^{(0)}=g, g^{(k+1)}=\left(g^{(k)}\right)^{\prime}$.

Напомним, что идеалом $A_{0}$ алгебры $A$ называется подалгебра алгебры $A$ такая, что коммутаторы элементов подалгебры с любым элементом алгебры являются элементами подалгебры. Легко доказывается следуюшее

УТВЕРЖДЕНИЕ 2.1.

1. Коммутант $g^{(1)}$ алгебры $g$ является идеалом, однако $g^{(2)}$ не является идеалом алгебры $g$.

2. Кратный коммутант $g^{(k+1)}$ является идеалом алгебры $g^{(k)}$, однако не является идеалом алгебры $g^{(l)}$, әде $l<k$.

3. Кратный коммутант $g^{(k+1)}$ является идеалом алгебры $g^{(l)}$, әде $l<k$, лишь при условии выполнения тождества Якоби.

Введем

ОПредЕлЕниЕ 2.4. Пусть $A^{(-)}$- коммутаторная алгебра, ассоциированная с некоторой алгеброй $A$. Алгебру $A^{(-)}$назовем коммутантно лиевой алгеброй, если любые три ее коммутатора порождают подалгебру, являюшуюся алгеброй Ли, т.е. если коммутант этой алгебры является лиевой подалгеброй.

Нетрудно увидеть, что коммутантно лиева алгебра является алгеброй Валя. 
2.2. Обобщенная алгебра Гейзенберга-Вейля и алгебра Валя. Рассмотрим некоторые свойства, которым должна удовлетворять обобшенная алгебра Гейзенберга-Вейля $W_{N}^{*}$, определенная в третьей части [3] работы.

ТЕОРЕМА 2.1. Обобщенная алгебра Гейзенберга-Вейля $W_{N}^{*}$ является алгеброй Валя, но не является алгеброй Ли, т.е. $W_{N}^{*}$-алгебра - нелиева алгебра Валя.

ДокАЗАТЕЛЬСТво. Используя соотношение для коммутаторов элементов $z_{n}$ и $z_{m}$ обобщенной алгебры Гейзенберга-Вейля, имеющее вид

$$
\left[z_{n}, z_{m}\right]=\imath s_{3}^{n m} I+\imath t_{k}^{n m} F_{k}(Q, P)
$$

где

$$
s_{3}^{n m}=x_{k}^{n} y_{k}^{m}-x_{k}^{m} y_{k}^{n}, \quad t_{k}^{n m}=t^{n} y_{k}^{m}-t^{m} y_{k}^{n},
$$

получаем выражение для якобиана коммутаторов

$$
J\left[\left[z_{1}, z_{2}\right],\left[z_{3}, z_{4}\right],\left[z_{5}, z_{6}\right]\right]=-i t_{k}^{12} t_{l}^{34} t_{m}^{56} J\left[F_{k}, F_{l}, F_{m}\right] .
$$

Поскольку операторы $F_{k}(Q, P)$ являются ассоциативными, получаем

$$
J\left[F_{k}, F_{l}, F_{m}\right]=0 .
$$

Таким образом, элементы обобщенной алгебры Гейзенберга-Вейля [3] удовлетворяют мягкому тождеству Якоби.

Можно показать, что обобщенная алгебра Гейзенберга-Вейля $W_{N}^{*}$ и ее подалгебры обладают следуюшими свойствами:

1. Алгебра Гейзенберга-Вейля $W_{N}$ является двусторонним идеалом обобщенной алгебры Гейзенберга-Вейля $W_{N}^{*}$, причем этот двусторонний идеал не является вполне простым в силу тождества $\left[W, Q_{i}\right]=0$.

2. Коммутант обобщенной алгебры Гейзенберга-Вейля является подалгеброй алгебры Гейзенберга-Вейля.

3. Максимальный двусторонний идеал обобщенной алгебры Гейзенберга-Вейля $W_{N}^{*}$, являющийся алгеброй Ли, совпадает с алгеброй Гейзенберга-Вейля $W_{N}$.

Рассмотрим наиболее простой пример коммутантно лиева обобщения алгебры Гейзенберга-Вейля $W_{N}$, а именно линейную обобщенную алгебру Гейзенберга-Вейля $L W_{N}^{*}$, определенную в [3]. Можно сформулировать

УТВеРЖДЕнИЕ 2.2. Обобщенная алгебра Гейзенберга-Вейля $L W_{N}^{*}$ удовлетворяет помимо мягкого тождества Якоби следующим более сильным тождествам:

$$
\begin{aligned}
J\left[\left[z_{1}, z_{2}\right],\left[z_{3}, z_{4}\right], z_{5}\right] & =0, \\
{\left[J\left[z_{1}, z_{2}, z_{3}\right], z_{4}\right] } & =0, \\
{\left[\left[z_{1}, z_{2}\right],\left[z_{3}, z_{4}\right]\right] } & =0 .
\end{aligned}
$$

Отметим, что соотношение (2) и следующее из него мягкое тохдество Якоби являются следствием тождества (4).

Для доказательства тождеств (2)-(4) достаточно использовать соотношение (1) и определение [3] алгебры $L W_{N}^{*}$. Коммутаторную алгебру, определяемую соотношением (4), назовем коммутантно коммутативной алгеброй. Такая алгебра является 
разрешимой [2], так как существует кратный коммутант, равный нулю алгебры, и, следовательно, цепочка кратных коммутантов обрывается.

В результате линейная обобщенная алгебра Гейзенберга-Вейля $L W^{*}$ является коммутантно коммутативной алгеброй, якобианы которой принадлежат центру данной алгебры. В силу разрешимости такой алгебры можно ввести понятие радикала, т.е. наибольшего разрешимого идеала, что облегчает изучение ее структурных свойств.

2.3. Коммутантно ассоциативная алгебра. Напомним, что алгебра называется ассоциативной алгеброй, если ассоциатор произвольных трех ее элементов равен нулю, т.е. операция умножения удовлетворяет условию $(x, y, z)=0$, где $(x, y, z) \equiv$ $(x y) z-x(y z)$ - ассоциатор операторов $x, y, z$. Введем следуюшие определения.

ОПреДЕЛЕниЕ 2.5. Неассоциативную алгебру назовем коммутантно ассоциативной алгеброй (или просто коммутантной алгеброй), если операция умножения удовлетворяет условию

$$
(x y-y x, z p-p z, q l-l q)=0 .
$$

ОПРЕДЕЛЕНИЕ 2.6. Неассоциативную алгебру назовем квазикоммутантной алгеброй, если операция умножения удовлетворяет условиям

1) левой квазикоммутантности: $(x y-y x, z p-p z, q)=0$;

$2)$ правой квазикоммутантности: $(q, x y-y x, z p-p z)=0$.

Видно, что квазикоммутантная алгебра является коммутантно ассоциативной. Обратное утверждение неверно. Кроме того, коммутаторная алгебра любой квазикоммутантной алгебры удовлетворяет тождеству (2). Сформулируем теорему.

Теорема 2.2. Коммутаторная алгебра $A^{(-)}$произвольной коммутантно ассоциативной алгебры $А$ является коммутантно лиевой алгеброй.

ДоКАЗАТЕЛЬСТВо теоремы основано на том, что алгебраический якобиан, определяемый для коммутаторной алгебры формулой

$$
J[A, B, C]=[[A, B], C]+[[B, C], A]+[[C, A], B],
$$

может быть записан через ассоциаторы в виде

$$
J[A, B, C]=(A, B, C)-(A, C, B)+(C, A, B)-(B, A, C)+(B, C, A)-(C, B, A) .
$$

Следовательно, используя определения 2.4. для коммутаторной алгебры некоторой коммутантно ассоциативной алгебры, можно получить искомое утверждение.

2.4. Обобщенная алгебра Гейзенберга-Вейля и ассоциатор. Как было показано в работе [8], обобщенную алгебру Гейзенберга-Вейля можно реализовать как коммутаторную алгебру некоторой неассоциативной алгебры.

Обозначим через $E_{k}=\left(Q_{k}, P_{k}\right)$ базисные элементы алгебры Гейзенберга-Вейля. Тогда для выполнения коммутационных соотношений обобщенной алгебры Гейзенберга-Вейля [3] достаточно, чтобы базисные элементы $\left(E_{k}, W\right)$ удовлетворяли соотношениям

$$
\begin{aligned}
& \left(E_{k}, E_{l}, E_{j}\right)=\left(E_{k}, E_{l}, W\right)=\left(W, E_{k}, E_{l}\right)=\left(E_{k}, W, E_{k}\right)=(W, W, W)=0, \\
& \left(E_{k}, W, E_{l}\right) \not \equiv 0, \quad\left(W, W, E_{k}\right) \not \equiv 0, \quad\left(E_{k}, W, W\right) \not \equiv 0, \quad\left(W, E_{k}, W\right) \not \equiv 0 .
\end{aligned}
$$

Используя соотношения (7), (8), легко доказать следуюшее 
УТВЕРЖДЕНИЕ 2.3. Неассоииативная алгебра, элементы которой представимы в виде $z=x_{k} E_{k}+t W$, где $x_{k}, t-$ числа, а базисные әлементы $\left(E_{k}, W\right)$ удовлетворяют соотношениям (7), (8), является квазикоммутативной алгеброй, т.е.

$$
\left(z_{1} z_{2}-z_{2} z_{1}, z_{3} z_{4}-z_{4} z_{3}, z_{5}\right)=\left(z_{5}, z_{1} z_{2}-z_{2} z_{1}, z_{3} z_{4}-z_{4} z_{3}\right)=0
$$

Кроме того, в силу соотношений (1) базисные элементы $\left(E_{k}, W\right)$ удовлетворяют соотношению коммутантной ассоциативности (5).

\section{3. ЛУПЫ С АССОЦИАТИВНЫМИ КОММУТАНТАМИ}

3.1. Лупы с обратимостью и коммутанты луп. Аналитическими лупами называются неассоциативные обобшения аналитических групп (групп Ли), впервые рассмотренные Мальцевым [5].

Множество $G$ с определенной на нем бинарной операцией умножения о называется квазигруппой, если для любых $a, b \in G$ каждое из уравнений $a \circ x=b$ и $y \circ a=b$ имеет единственное решение. Если в квазигруппе $G$ сушествует такой элемент $e \in G$ (единица), что $e \circ x=x \circ e=x$ для всех $x \in G$, то квазигруппа называется лупой [4].

Пусть $G$ - лупа, т.е. множество элементов $G$ с определенными на нем операциями умножения, правого и левого деления $(\circ, /, \backslash)$ такое, что существует элемент $e \in G$, для которого $x \circ e=e \circ x=x$ и $(x / y) \circ y=y \circ(y \backslash x)=(x \circ y) / y=y \backslash(y \circ x)=x$ для всех $x$ и $y$ из $G$.

Хотя лупа обладает единицей $e$ и, будучи квазигруппой, для всякого элемента $a$ содержит элементы $e / a$ и $e \backslash a$, однако эти элементы не являются обратными элементами произвольной лупы. Рассмотрим поэтому следующий более узкий класс луп. Лупа $G$ называется лупой с обратимостью, если для любых двух элементов $a, b \in G$ имеют место равенства

$$
(b \circ a) \circ(e / a)=b, \quad(e \backslash a) \circ(a \circ b)=b .
$$

Если подставить в первое из равенств вместо $b$ элемент $e / a$, то получим $e / a=e \backslash a$. Таким образом, всякий элемент лупы с обратимостью обладает однозначно определенным двусторонним обратным элементом $a^{-1}$ :

$$
a \circ a^{-1}=a^{-1} \circ a=e
$$

Равенства (9), определяющие лупу с обратимостью, принимают теперь вид

$$
(b \circ a) \circ a^{-1}=a^{-1} \circ(a \circ b)=b .
$$

Отметим, что для луп с обратимостью выполняется важное тождество

$$
(a \circ b)^{-1}=b^{-1} \circ a^{-1}
$$

На самом деле, если $a \circ b=c$, то

$$
b=a^{-1} \circ c \Rightarrow a^{-1}=b \circ c^{-1} \Rightarrow c^{-1}=b^{-1} \circ a^{-1} .
$$

Можно определить понятие коммутатора и коммутанта лупы. 
Коммутатором двух элементов $x$ и $y$ лупы $G$ называется элемент $z$ вида

$$
z=[x, y]=(x \circ y) \circ(y \circ x)^{-1}
$$

В лупах с обратимостью коммутатор двух элементов $x$ и $y$ в силу тождества (12) можно представить в виде $[x, y]=(x \circ y) \circ\left(x^{-1} \circ y^{-1}\right)$.

Коммутантом лупы $G$ называется множество $G^{(-)}$всех элементов $z \in G$ лупы $G$, которые представляются в виде $z=z_{1} \circ z_{2} \circ \cdots \circ z_{m}$, где каждое $z_{i}$ является коммутатором двух элементов $x_{i}, y_{i} \in G$, т.е. $z_{i}=\left(x_{i} \circ y_{i}\right) \circ\left(y_{i} \circ x_{i}\right)^{-1}$.

3.2. Касательная алгебра аналитической лупы. Аналитической лупой называется аналитическое многообразие, снабженное структурой лупы такой, что операция умножения является аналитической $[5,2]$.

Касательной алгеброй $g$ локальной аналитической лупы $G$ называется касательное пространство $T_{e}(G)$, в котором определены бинарная и тернарная операции [, ] и $\langle,$,$\rangle следующим образом. Пусть \alpha(t), \beta(t), \gamma(t)$ - дифференцируемые пути в лупе $G$, удовлетворяющие условиям $\alpha(0)=\beta(0)=\gamma(0)=e, \alpha^{\prime}(0)=\xi, \beta^{\prime}(0)=\eta, \gamma^{\prime}(0)=\zeta$. Тогда

$$
\begin{aligned}
(\beta(t) \circ \alpha(t)) \backslash(\alpha(t) \circ \beta(t)) & =t^{2}[\xi, \eta]+o\left(t^{2}\right), \\
(\alpha(t) \circ(\beta(t) \circ \gamma(t))) \backslash((\alpha(t) \circ \beta(t)) \circ \gamma(t)) & =t^{3}\langle\xi, \eta, \zeta\rangle+o\left(t^{3}\right)
\end{aligned}
$$

В локальной системе координат на аналитической лупе $G$ в окрестности единишы произведение двух элементов $z_{i}=\mu_{i}(x, y)$ можно разложить в ряд Тейлора

$$
\mu_{i}(x, y)=x_{i}+y_{i}+a_{j k}^{i} x_{j} y_{k}+b_{j k l}^{i} x_{j} x_{k} y_{l}+c_{j k l}^{i} x_{j} y_{k} y_{l}+\cdots
$$

В силу сушествования единичного элемента в лупе функции $\mu_{i}(x, y)$, как и в случае групп Ли, обладают свойством

$$
\left[\frac{\partial^{r} \mu_{i}}{\partial x_{i_{1}} \ldots \partial x_{i_{r}}}\right]_{x=0, y=0}=0, \quad\left[\frac{\partial^{r} \mu_{i}}{\partial y_{i_{1}} \ldots \partial y_{i_{r}}}\right]_{x=0, y=0}=0 .
$$

В локальной системе координат бинарная и тернарная операции имеют вид

$$
[\xi, \eta]_{i}=u_{j k}^{i} \xi^{j} \eta^{k}, \quad\langle\xi, \eta, \zeta\rangle_{i}=v_{j k l}^{i} \xi^{j} \eta^{k} \zeta^{l}
$$

где

$$
u_{j k}^{i}=a_{j k}^{i}-a_{k j}^{i}, \quad v_{j k l}^{i}=2 b_{j k l}^{i}-2 c_{j k l}^{i}+\frac{1}{4} u_{m l}^{i} v_{j k}^{m}-\frac{1}{4} v_{j m}^{i} v_{k l}^{m} .
$$

Если локальная лупа ассоциативна, т.е. является группой Ли, то тернарная операция тождественно равна нулю $\langle\xi, \eta, \zeta\rangle=0$ для всех $\xi, \eta, \zeta \in g$. Для диассоциативных (альтернативных) локальных аналитических луп (лупа, любая пара элементов которой порождает ассоциативную подлупу) тернарная операция в касательной алгебре выражается через бинарную по формуле

$$
\langle\xi, \eta, \zeta\rangle=-\frac{1}{6} J[\xi, \eta, \zeta]
$$


где

$$
J[\xi, \eta, \zeta]=[[\xi, \eta], \zeta]+[[\eta, \zeta], \xi]+[[\zeta, \xi], \eta] .
$$

Таким образом, для произвольной лупы в канонических координатах имеет место формула, связывающая операцию умножения в лупе и операцию умножения в касательной алгебре $x \circ y=x+y+(1 / 2) x y+\cdots$, где $x y$ - бинарная антикоммутативная (т.е. $x x=0$, или, что то же самое, $x y=-y x$ ) операция умножения соответствующих элементов касательной алгебры данной лупы. Покажем, что коммутатор лупы выражается в канонических координатах в виде

$$
[x, y] \equiv(x \circ y) \circ(y \circ x)^{-1}=x y+\cdots,
$$

где многоточием обозначены элементы степени, большей либо равной трем. Действительно, используя формулы

$$
x \circ y=x+y+(1 / 2) x y+\cdots, \quad(y \circ x)^{-1}=-y-x-(1 / 2) y x-\cdots,
$$

получаем для коммутатора двух элементов лупы

$$
[x, y]=(x \circ y) \circ(y \circ x)^{-1}=(1 / 2)(x y-y x+x x-x y-y x+y y)+\cdots .
$$

И если бинарная операция в касательной алгебре является антикоммутативной $(x x=0$ и $x y=-y x)$, то приходим к искомому соотношению (20).

3.3. Касательная алгебра коммутантно ассоциативной лупы. Введем следуюшее

ОПРЕДЕЛЕНИЕ 3.1. Коммутантно ассоциативной лупой (лупой Валя) назовем лупу с обратимостью, коммутант которой является ассоциативной подлупой (т.е. группой).

Классическое соответствие между аналитическими локальными группами (группами Ли) и алгебрами Ли, устанавливаемое теоремами Ли [1], имеет место и между аналитическими локальными коммутантно ассоциативными лупами с обратимостью (лупами Валя) и коммутантно лиевыми алгебрами (алгебрами Валя).

ТЕОрема 3.1. А. Касательная алгебра аналитической локальной коммутантно ассоциативной лупы является коммутантно лиевой алгеброй.

Б. Всякая конечная коммутантно лиева алгебра есть касательная алгебра одной и (с точностью до локальных изоморфизмов) только одной локальной аналитической коммутантно ассоииативной лупь.

ДокАЗАТЕльСтво.А.Рассмотрим произвольный элемент коммутанта лупы $G$ с обратимостью. По определению коммутанта он представим в виде $z_{1} \circ z_{2} \circ \cdots \circ z_{m}$, где каждое $z_{k}$ является коммутатором двух элементов $x_{k}$ и $y_{k}$ лупы $G$. Если лупа $G$-коммутантно ассоциативная лупа, то произведение вида $g_{1}\left(t_{1}\right) \circ g_{2}\left(t_{2}\right) \circ \cdots \circ g_{m}\left(t_{m}\right)$, где $g_{k}\left(t_{k}\right)$ являются произвольными однопараметрическими подгруппами с касательными векторами $z_{k}$, не зависит при малых $t_{k}$ от расстановки скобок. Следовательно, подалгебра касательной алгебры, порожденная элементами $z_{k}$, является лиевой алгеброй, т.е. сама алгебра является коммутантно лиевой. 
Б. Введем координаты первого рода и заметим, что однопараметрические подгруппы $g_{k}\left(t_{k}\right)$ порождают ассоциативную локальную подлупу. Видно, что в лупе в канонических координатах имеет место разложение в ряд Тейлора, позволяющий по алгебре восстановить лупу.

Доказательство утверждения Б в обшем случае является в настоящий момент открытым вопросом.

\section{4. ПРЕДСТАВЛЕНИЕ КОММУТАНТНО ЛИЕВОЙ АЛГЕБРЫ КАК АЛГЕБРЫ ДИФФЕРЕНЦИАЛЬНЫХ ФОРМ}

4.1. Алгебра Пуассона для дифференциальных 1-форм. Известно, что симплектическим многообразием $\left(M^{2 n}, \omega\right)$ назьвается дифференцируемое многообразие $M^{2 n}$ четной размерности $2 n$, на котором определена дифференциальная форма $\omega$ степени 2 , являюшаяся замкнутой $(d \omega=0)$ и невырожденной.

На симплектическом многообразии можно определить для замкнутых дифференциальных 1-форм скобку Пуассона $[9,10]$. Скобкой Пуассона двух замкнутых дифференциальных 1-форм $\alpha=a_{k}(z) d z^{k}$ и $\beta=b_{k}(z) d z^{k}$ на симплектическом многообразии $(M, \omega)$ называется точная 1 -форма $(\alpha, \beta)$, определенная по правилу

$$
(\alpha, \beta)=d \Psi(\alpha, \beta)=d \omega\left(X_{\alpha}, X_{\beta}\right),
$$

где

$$
\Psi(\alpha, \beta)=\omega\left(X_{\alpha}, X_{\beta}\right)=\Psi^{k l} a_{k} b_{l}, \quad d \alpha=d \beta=0
$$

и $X_{\alpha}$ - векторное поле, которое связано с соответствуюшей 1-формой $\alpha$ по правилу $i\left(X_{\alpha}\right) \omega=i_{X_{\alpha}} \omega=\alpha ; \omega$ - замкнутая невырожденная 2-форма $[9,10]$, называемая симплектической формой; $i$ - внутреннее умножение векторных полей и дифференциальных форм [9]; $\Psi$ - косимплектическая структура, а $\Psi^{k l}$ - матрица, являющаяся обратной к матрице симплектической формы и удовлетворяюшая условиям [10]

а) антисимметрии: $\Psi^{k l}=-\Psi^{l k}$;

б) обрашения в нуль скобок Схоутена:

$$
[\Psi, \Psi]^{s l k}=\Psi^{s m} \partial_{m} \Psi^{l k}+\Psi^{l m} \partial_{m} \Psi^{k s}+\Psi^{k m} \partial_{m} \Psi^{s l}=0 .
$$

Если эта билинейная операция “скобка Пуассона" определена на пространстве замкнутых 1-форм $\Lambda_{0}^{1}(M)$, то многообразие $M$ называется пуассоновым многообразием, а пространство $\Lambda_{0}^{1}(M)$ с этой билинейной операцией - алгеброй Пуассона $P_{1}$. Алгебра Пуассона $P_{1}$ является алгеброй Ли. Это следует из выполнения условия антикоммутативности $(\alpha, \beta)=-(\beta, \alpha)$ и тождества Якоби

$$
J(\alpha, \beta, \gamma)=((\alpha, \beta), \gamma)+((\beta, \gamma) \alpha)+((\gamma, \alpha), \beta)=0
$$

Если распространить определение (21) на произвольные, в том числе незамкнутые, дифференциальные 1-формы, то для незамкнутых 1-форм тождество Якоби не выполняется, $J(\alpha, \beta, \gamma) \not \equiv 0$. Поэтому обобшение алгебры Пуассона, т.е. пространство дифференциальных 1-форм $\Lambda^{1}(M)$ с такой билинейной операцией, не является алгеброй Ли.

В ряде работ $[10,9]$ рассматривается обобщение скобки Пуассона на незамкнутые дифференциальные формы, которое удовлетворяет тождеству Якоби. В этом случае 
алгебра Пуассона незамкнутых дифференциальных 1-форм является алгеброй Ли. Например, можно определить скобку Пуассона двух дифференциальных 1-форм $\alpha=a_{k}(z) d z^{k}$ и $\beta=b_{k}(z) d z^{k}$ на симплектическом многообразии $(M, \omega)$ как 1-форму $(\alpha, \beta)$, задаваемую правилом [10]

$$
(\alpha, \beta)=d \Psi(\alpha, \beta)+\Psi(d \alpha, \beta)+\Psi(\alpha, d \beta)
$$

Эта скобка Пуассона удовлетворяет условию антикоммутативности и тождеству Якоби (23). Поэтому алгебра Пуассона незамкнутых дифференциальных форм со скобкой вида (24) является лиевой алгеброй.

4.2. Незамкнутые формы и диссипативные системы. Заметим, что негамильтоновость и диссипативные свойства динамических систем связаны со свойствами незамкнутых дифференциальных форм.

Следуя [9], гамильтоновой системой на симплектическом многообразии $\left(M^{2 n}, \omega\right)$ мы будем называть векторное поле $X$ на $M^{2 n}$ такое, что дифференциальная 1-форма $i_{X} \omega$ замкнута.

Если форма $i_{X} \omega$ точна, то существует дифференцируемая функция $H$ на $M^{2 n}$, называемая гамильтонианом системы, такая, что $i_{X} \omega=-d H$.

Известно, что система, задаваемая векторным полем $X$ на симплектическом многообразии $\left(M^{2 n}, \omega\right)$, является гамильтоновой системой тогда и только тогда, когда лиева производная по векторному полю от симплектической формы равна нулю, $L_{X} \omega=0$, где $L_{X}=d i_{X}+i_{X} d-$ лиева производная по векторному полю $X$.

Введем следуюшее очевидное

ОПРЕДЕлЕНИЕ 4.1. Диссипативной (негамильтоновой) системой на симплектическом многообразии $\left(M^{2 n}, \omega\right)$ называется векторное поле $X$ на $M^{2 n}$ такое, что дифоференциальная 1-форма $i_{X} \omega$ незамкнута.

Таким образом, построение коммутантно лиевой алгебры незамкнутых дифференциальных форм и соответствуюших им векторных полей позволяет описывать свойства классических диссипативных систем на симплектическом многообразии.

4.3. Обобшенная алгебра Пуассона для дифференциальных форм. Для описания классических диссипативных систем предлагается обобщить алгебру Пуассона $P_{1}$. Определим билинейную операцию на $\Lambda^{1}(M)$.

ОПРЕДЕЛЕНИЕ 4.2. Обобщенной скобкой Пуассона для двух 1 -форм $\alpha$ и $\beta$ называется точная дифференциальная 1-форма $[\alpha, \beta]$, определенная по правилу

$$
[\alpha, \beta]=d \Psi(\alpha, \beta)=d \omega\left(X_{\alpha}, X_{\beta}\right)
$$

Данное определение получается прямым обобшением скобки Пуассона (21) на незамкнутые формы. Скобка Пуассона (25) для незамкнутых 1-форм не удовлетворяет тождеству Якоби,

$$
J[\alpha, \beta, \gamma]=[[\alpha, \beta], \gamma]+[[\beta, \gamma], \alpha]+[[\gamma, \alpha], \beta] \not \equiv 0 .
$$

Поэтому обобшенная алгебра Пуассона $P_{1}^{*}$ не является алгеброй Ли. Тождество Якоби выполняется для замкнутых 1-форм. Следовательно, замкнутые 1-формы определяют 
алгебру Ли, которая есть алгебра Пуассона $P_{1}$. В результате обобшенная алгебра Пуассона $P_{1}^{*}$ содержит подалгебру, являющуюся алгеброй Пуассона. Обобщенная скобка Пуассона двух незамкнутых 1-форм есть замкнутая 1-форма, поэтому подалгебра $P_{1}$ является двусторонним идеалом алгебры $P_{1}^{*}$, и, следовательно, сушествует точная диаграмма:

$$
0 \rightarrow P_{1} \rightarrow P_{1}^{*} \rightarrow P_{1}^{*} / P_{1} \rightarrow 0 .
$$

Обобшенная скобка Пуассона для 1-форм удовлетворяет следуюшим свойствам:

1) антикоммутативности: $\forall \alpha, \beta \in P_{1}^{*}[\alpha, \beta]=-[\beta, \alpha] \in P_{1}$;

2) тождеству Якоби: $\forall \alpha, \beta, \gamma \in P_{1} \quad J[\alpha, \beta, \gamma]=0$;

$3)$ нелиевости: $\forall \alpha, \beta, \gamma \in P_{1}^{*} \quad J[\alpha, \beta, \gamma] \not \equiv 0$;

4) дистрибутивности: $\forall \alpha, \beta, \gamma \in P_{1}^{*} \quad[a \alpha+b \beta, \gamma]=a[\alpha, \gamma]+b[\beta, \gamma]$, где $a$ и $b-$ действительные числа.

Легко доказать следуюшую теорему.

Теорема 4.1. Обобщенная алгебра Пуассона является коммутантно лиевой алгеброй, т.е. обобщенная скобка Пуассона (25) удовлетворяет условию антикоммутативности $[\alpha, \alpha]=0$ и мягкому тождеству Якоби:

$$
J[[\alpha, \beta],[\gamma, \delta],[\mu, \nu]]=0 .
$$

Таким образом, структура коммутантно лиевой алгебры, которая не является алгеброй Ли, естественно определяется на пространстве дифференциальных 1-форм $\Lambda^{1}(M)$ на симплектическом многообразии $M$. Коммутантно лиева алгебра дифференциальных форм является обобщением алгебры Ли замкнутых форм и содержит данную алгебру Ли в качестве своей подалгебры (идеала).

4.4. Обобщенная алгебра Пуассона для векторных полей. Аналогично алгебре Пуассона для дифференциальных форм можно обобшить алгебру Пуассона для векторных полей. Введем

ОПРЕДЕЛЕнИЕ 4.3. Обобщенной скобкой Пуассона для двух векторных полей $X$ и $Y$ будем называть векторное поле $\{X, Y\}$, определенное по правилу

$$
\{X, Y\}=Z_{d \omega(X, Y)},
$$

где $d \omega(X, Y)=d \Psi\left(i_{X} \omega, i_{Y} \omega\right)$, а $Z_{\alpha}$ - векторное поле, соответствуюшее дифференциальной 1-форме $\alpha$ по следуюшему правилу: $i_{Z_{\alpha}} \omega=\alpha$.

Известно [9], что если векторным полям $X$ и $Y$ соответствуют замкнутые дифференциальные формы, т.е. $d\left(i_{X} \omega\right)=d\left(i_{X} \omega\right)=0$, то обобщенная скобка Пуассона для этих векторных полей совпадает с коммутатором: $\{X, Y\}=-[X, Y]$. Для доказательства этого утверждения необходимо воспользоваться тождествами

$$
Z_{i_{X} \omega}=X \text { и } d \omega(X, Y)=-i_{[X, Y]} \omega-i_{Y} d\left(i_{X} \omega\right)+i_{X} d\left(i_{Y} \omega\right),
$$

где $[X, Y]=X Y-Y X-$ коммутатор. Тогда легко получить соотношение

$$
\{X, Y\}=Z_{d \omega(X, Y)}=Z_{i_{[X, Y]}} \omega=-[X, Y] .
$$


В общем случае взаимосвязь обобщенных скобок Пуассона и коммутаторов векторных полей имеет вид

$$
\{X, Y\}=-[X, Y]-Z_{\beta(X, Y)},
$$

где $\beta(X, Y)=i_{Y} d\left(i_{X} \omega\right)-i_{X} d\left(i_{Y} \omega\right)$.

Можно увидеть, что тождество Якоби для обобщенных скобок Пуассона векторных полей не выполняется, $J\{X, Y, Z\} \not \equiv 0$. Однако тождество Якоби выполняется для векторных полей, соответствуюшие дифференциальные 1-формы которых замкнуты. Такие векторные поля определяют лиеву алгебру, которая есть алгебра Пуассона с коммутатором в качестве билинейной операции. Следовательно, верна следуюшая

Теорема 4.2. Обобщенная алгебра Пуассона для векторных полей является коммутантно лиевой алгеброй, т.е. обобщенная скобка Пуассона (27) удовлетворяет условию антикоммутативности $\{X, Y\}=-\{Y, X\}$ и мягкому тождеству Якоби $J\{\{X, Y\},\{Z, K\},\{L, F\}\}=0$.

Таким образом, структура коммутантно лиевой алгебры естественно определяется для пространства векторных полей $T(M)$. Эта алгебра является обобшением алгебры Ли векторных полей и содержит данную лиеву алгебру в качестве своей подалгебры (идеала). В силу определения 4.1 векторные поля, соответствуюшие незамкнутым дифференциальным формам, описывают классические диссипативные системы. Поэтому свойства построенной коммутантно лиевой алгебры связаны со свойствами классических диссипативных систем.

\section{5. БЕСКОНЕЧНЫЕ МАТРИЦЫ}

Сделаем несколько замечаний относительно другого примера коммутантно лиевой алгебры. Известно, что наблюдаемые величины в квантовой теории можно описывать бесконечными матрицами. Бесконечные матрицы в квантовой механике гамильтоновых систем должны удовлетворять перестановочным соотношениям Гейзенберга. Кроме того, на них накладывается дополнительное условие в виде тождества Якоби, что приводит к ассоциативности бесконечных матриц. Поэтому для описания квантовых гамильтоновых систем обычно используются бесконечные матришы Гильберта. Для решения проблем квантового описания диссипативных систем необходимо использовать операторы, которые нарушают тождество Якоби, что приводит к отказу от свойства ассоциативности для бесконечных матриц, соответствуюших этим операторам.

Известно, что в общем случае умножение бесконечных матриц не ассоциативно,

$$
(A B) C \not \equiv A(B C) \quad \text { или } \quad \sum_{l=1}^{\infty}\left(\sum_{k=1}^{\infty} a_{i k} b_{k l}\right) c_{l j} \not \equiv \sum_{k=1}^{\infty} a_{i k}\left(\sum_{l=1}^{\infty} b_{k l} c_{l j}\right) .
$$

Это легко показать на следуюшем примере [11]. Пусть $a_{k l}=c_{k l}=1$ для любых $k$ и $l$ и верно неравенство

$$
\sum_{l=1}^{\infty}\left(\sum_{k=1}^{\infty} b_{k l}\right) \not \equiv \sum_{k=1}^{\infty}\left(\sum_{l=1}^{\infty} b_{k l}\right),
$$

тогда $(A B) C \not \equiv A(B C)$, ибо $(A B) C$ и $A(B C)$ равны соответственно левой и правой частям (32). В качестве примера матришы, удовлетворяюшей условию (32), можно взять 
матрицу $\left(b_{k l}\right)$, в которой

$$
\begin{gathered}
b_{k l}=\frac{(k-l)}{2^{k+l-2}} \frac{(k+l-3) !}{(k-1)(l-1)} \quad(k>1, \quad l>1), \\
b_{k 1}=2^{-(k-1)}(k>1), \quad b_{1 l}=-2^{-(l-1)} \quad(l>1), \quad b_{11}=0 .
\end{gathered}
$$

В этом случае

$$
\sum_{l=1}^{\infty}\left(\sum_{k=1}^{\infty} b_{k l}\right)=1, \quad \sum_{k=1}^{\infty}\left(\sum_{l=1}^{\infty} b_{k l}\right)=-1 .
$$

Напомним, что бесконечная матрица $A=\left(a_{p q}\right)$ является матрицей Гильберта, если существует билинейная форма

$$
A(x, y)=\sum_{p=1}^{\infty} \sum_{q=1}^{\infty} x_{p} a_{p q} y_{q}
$$

как внутренний предел на единичной гиперсфере [11].

ОПРЕДЕЛЕнИЕ 5.1. Матрицей Гильберта называется бесконечная матрица $A=\left(a_{p q}\right)$, если для любого $\varepsilon>0$ и любой пары $x=\left(x_{p}\right)$ и $y=\left(y_{q}\right)$ единичных векторов, которые удовлетворяют условию

$$
\sum_{p=1}^{\infty}\left|x_{p}\right|^{2}=\sum_{q=1}^{\infty}\left|y_{q}\right|^{2}=1
$$

существует число $A(x, y)$, не зависимое от $\varepsilon$, и два числа $M(\varepsilon)$ и $N(\varepsilon)$ такие, что

$$
\left|A(x, y)-\sum_{p=1}^{m} \sum_{q=1}^{n} x_{p} a_{p q} y_{q}\right|<\varepsilon
$$

всякий раз, когда $m>M(\varepsilon)$ и $n>N(\varepsilon)$.

Отметим, что для двух произвольных бесконечных матриц $A=\left(a_{p q}\right)$ и $B=\left(b_{p q}\right)$ из сушествования произведения $A B$ не следует сушествования произведения $B A$. Например, если $a_{i j}=0(j>1)$, то

$$
(B A)_{i j}=\sum_{k=1}^{\infty} a_{i k} b_{k j}=0 \quad(j>1), \quad(B A)_{i j}=\sum_{k=1}^{\infty} a_{i k} b_{k 1} \quad(j=1) .
$$

Отсюда следует, что $B A$ не сушествует, если ряды $\sum_{k=1}^{\infty} a_{i k} b_{k 1}$ расходятся.

Введем следуюшее

ОПРЕДЕЛЕНИЕ 5.2. Диссипативными матрицами называются бесконечные матрицы, для которых всегда сушествуют произведения этих матриц друг на друга и коммутатор произвольных двух матрищ $A=\left(a_{p q}\right)$ и $B=\left(b_{p q}\right)$ равен некоторой матрище Гильберта $H=\left(h_{p q}\right)$, т.е. $A B-B A=H$.

Видно, что в общем случае диссипативные матришы не являются ассоциативными и не удовлетворяют тождеству Якоби. Однако верна 
ТЕОРема 5.1. Диссипативные матрииы удовлетворяют мягкому тожсдеству Якоби и, следовательно, образуют коммутантно лиеву алгебру.

Для доказательства первого утверждения достаточно воспользоваться свойством ассоциативности матриц Гильберта и представлением якобиана через ассоциаторы, использованным в теореме 4.2 .

Поскольку сумма и разность диссипативных матриц являются диссипативными матрицами, то выполняется закон дистрибутивности для этих матриц и, следовательно, они образуют некоторую алгебру. В силу того что для коммутаторов данных матриц вьполнено условие антикоммутативности и мягкое тождество Якоби, диссипативные матрицы образуют коммутантно лиеву.

Заметим, что для произвольных бесконечных матриц правило дистрибутивности не имеет места. Это обусловлено тем, что произведение $A(B+C)$ может сушествовать, в то время как $A B$ и $A C$ не существуют. Например, такой случай имеет место для матриц $\left(a_{i j}\right),\left(b_{i j}\right)$ и $\left(c_{i j}\right)$, где $a_{i j}=1, b_{i j}=d_{i}+1, c_{i j}=d_{i}-1$ для любого $j$, и ряд $\sum_{i=1}^{\infty} d_{i}$ сходится.

Таким образом, алгебры некоторых бесконечных матриц не являются ассоциативными, что позволяет строить антикоммутативные нелиевы алгебры. Для описания эволюции квантовых диссипативных систем, видимо, можно использовать неассоциативные бесконечные матрицы, коммутаторы которых являются ассоциативными бесконечными матрицами (например, матрицами Гильберта). Однако описание свойств таких бесконечных неассоциативных матриц является в настоящий момент открытым вопросом.

\section{6. ЗАКЛЮЧЕНИЕ}

Сформулируем основные выводы.

1. Для непротиворечивого описания динамики квантовых диссипативных (негамильтоновых) систем необходимо отказаться от тождеств Якоби и рассматривать антикоммутативные нелиевы алгебры.

2. Аналогом алгебры Ли для квантовых диссипативных систем является коммутантно лиева алгебра (алгебра Валя), т.е. антикоммутативная алгебра, коммутаторы которой порождают лиеву подалгебру (или, другими словами, алгебра, коммутант которой является алгеброй Ли).

3. Аналогом группы Ли для квантовых диссипативных систем является лупа Валя, т.е. лупа, коммутант которой есть ассоциативная подлупа (группа).

Перечислим примеры коммутантно лиевых алгебр, приведенные в данной работе:

1) обобщенная алгебра Гейзенберга-Вейля (теорема 2.1);

2) коммутаторная алгебра, ассоциированная с коммутантно ассоциативной алгеброй (определение 2.5, теорема 2.2);

3) касательная алгебра аналитической коммутантно ассоциативной лупы (определение 3.1 , теорема 3.1 );

4) обобщенная алгебра Пуассона дифференциальных 1-форм и векторных полей (определения 4.2 и 4.3 , теоремы 4.2 и 4.3$)$;

5) алгебра бесконечных диссипативных матриц, коммутаторы которых являются ассоциативными матрицами (определение 5.2, теорема 5.1).

Указанные алгебры связаны с классическими и квантовыми негамильтоновыми (диссипативными) системами и, следовательно, интересны не только с математической, но 
и с физической точки зрения.

Работа выполнена при поддержке Российского фонда фундаментальных исследований, проект № 96-02-16413-а.

\section{Список литературы}

[1] Л. С. Понтрягин. Непрерывные группы. 3-е изд. М.: Наука, 1973.

[2] Общая алгебра. Т. 1. /Ред. Л. А. Скорняков. М.: Наука, 1990.

[3] B. E. Тарасов. ТМФ. 1997. Т. 110. № 1. С. 73-85.

[4] В. Д. Белоусов. Основы теории квазигрупп и луп. М.: Наука, 1967.

[5] А. И. Мальчев. Мат. сб. 1995. Т. 36. № 3. С. 569-573; Избранные труды. Т. 1. Классическая алгебра. М.: Наука, 1976. С. 340-345.

[6] К.А. Жевалков, А.М. Слинько, И. П. Шестаков, А. И. Шириов. Кольца, близкие к ассоциативным. М.: Наука, 1978.

[7] R. D. Shafer. An introduction to nonassociative algebras. London-New York: Academic Press, 1966.

[8] B. Е. Тарасов. ТМФ. 1994. Т. 100. № 3. С. 402-417.

[9] K. Годбийон. Дифференциальная геометрия и аналитическая механика. М.: Мир, 1973.

[10] М. В. Карасев, В. П. Маслов. Нелинейные скобки Пуассона. Геометрия и квантование. М.: Наука, 1991.

[11] P. Кук. Бесконечные матрицы и пространства последовательностей. М.: Наука, 1960.

Поступила в редакцию 30.IV.1996 г.

\section{V.E. Tarasov \\ QUANTUM DISSIPATIVE SYSTEMS. IV. ANALOG OF LIE ALGEBRA AND LIE GROUP}

The requirement of consistent quantum description of dissipative systems leads to necessity to go beyond Lie algebra and group. In order to describe dissipative (non-Hamiltonian) systems in quantum theory we need to use non-Lie algebra (algebras for which the Jacoby identity is not satisfied) and analytic quasigroups (nonassociative generalization of analytic groups). We prove that this analog is a commutant Lie algebra (an algebra, the commutant of which is a Lie subalgebra) and a commutant associative loop (a loop, commutators of which form an associative subloop (group)). We prove that the tangent algebra of an analytic commutant associative loop (Valya loop) is a commutant Lie algebra (Valya algebra). Examples of commutant Lie algebras are considered. 